ORIGINAL ARTICLE

\section{Coronary-Artery Bypass Surgery in Patients with Left Ventricular Dysfunction}

\author{
Eric J. Velazquez, M.D., Kerry L. Lee, Ph.D., Marek A. Deja, M.D., Ph.D., \\ Anil Jain, M.D., George Sopko, M.D., M.P.H., Andrey Marchenko, M.D., Ph.D., \\ Imtiaz S. Ali, M.D., Gerald Pohost, M.D., Sinisa Gradinac, M.D., Ph.D., \\ William T. Abraham, M.D., Michael Yii, M.S., F.R.C.S., F.R.A.C.S., \\ Dorairaj Prabhakaran, M.D., D.M., Hanna Szwed, M.D., Paolo Ferrazzi, M.D., \\ Mark C. Petrie, M.D., Christopher M. O'Connor, M.D., \\ Pradit Panchavinnin, M.D., Lilin She, Ph.D., Robert O. Bonow, M.D., \\ Gena Roush Rankin, M.P.H., R.D., Robert H. Jones, M.D., \\ and Jean-Lucien Rouleau, M.D., for the STICH Investigators*
}

A BSTRACT

BACKGROUND

The role of coronary-artery bypass grafting (CABG) in the treatment of patients with coronary artery disease and heart failure has not been clearly established.

METHODS

Between July 2002 and May 2007, a total of 1212 patients with an ejection fraction of $35 \%$ or less and coronary artery disease amenable to CABG were randomly assigned to medical therapy alone (602 patients) or medical therapy plus CABG (610 patients). The primary outcome was the rate of death from any cause. Major secondary outcomes included the rates of death from cardiovascular causes and of death from any cause or hospitalization for cardiovascular causes.

RESULTS

The primary outcome occurred in 244 patients (41\%) in the medical-therapy group and $218(36 \%)$ in the CABG group (hazard ratio with CABG, 0.86; 95\% confidence interval [CI], 0.72 to $1.04 ; \mathrm{P}=0.12)$. A total of 201 patients $(33 \%)$ in the medicaltherapy group and 168 (28\%) in the CABG group died from an adjudicated cardiovascular cause (hazard ratio with CABG, $0.81 ; 95 \% \mathrm{CI}, 0.66$ to $1.00 ; \mathrm{P}=0.05$ ). Death from any cause or hospitalization for cardiovascular causes occurred in 411 patients $(68 \%)$ in the medical-therapy group and 351 (58\%) in the CABG group (hazard ratio with CABG, 0.74 ; $95 \% \mathrm{CI}, 0.64$ to 0.85 ; $\mathrm{P}<0.001$ ). By the end of the followup period (median, 56 months), 100 patients in the medical-therapy group (17\%) underwent $\mathrm{CABG}$, and 555 patients in the CABG group (91\%) underwent CABG.

CONCLUSIONS

In this randomized trial, there was no significant difference between medical therapy alone and medical therapy plus CABG with respect to the primary end point of death from any cause. Patients assigned to CABG, as compared with those assigned to medical therapy alone, had lower rates of death from cardiovascular causes and of death from any cause or hospitalization for cardiovascular causes. (Funded by the National Heart, Lung, and Blood Institute and Abbott Laboratories; STICH ClinicalTrials.gov number, NCT00023595.)
The authors' affiliations are listed in the Appendix. Address reprint requests to Dr. Velazquez at Duke Clinical Research Institute, Rm. 0311 Terrace Level, 2400 Pratt St., Durham, NC 27705, or at eric. velazquez@duke.edu.

*A complete list of investigators participating in the hypothesis 1 component of the Surgical Treatment for Ischemic Heart Failure (STICH) trial is provided in the Supplementary Appendix, available at NEJM.org.

This article (10.1056/NEJMoal100356) was published on April 4, 2011, at NEJM.org.

N Engl J Med 2011;364:1607-16. Copyright @ 2011 Massachusetts Medical Society. 
T IS ESTIMATED THAT 5.8 MILLION PATIENTS in the United States ${ }^{1}$ and 15 million in Europe ${ }^{2}$ have heart failure. Coronary artery disease is the most common substrate for heart failure in industrialized nations. ${ }^{3}$ However, the role of coronary-artery bypass grafting (CABG) in the treatment of patients with coronary artery disease and heart failure has not been clearly established.

In three landmark clinical trials in the 1970s, a total of 2234 patients with chronic stable angina were randomly assigned to undergo CABG or receive medical therapy alone. ${ }^{4-6}$ The findings from these trials led to recommendations supporting the use of CABG to relieve disabling symptoms of angina, particularly among highrisk subgroups with extensive coronary artery disease. ${ }^{7,8}$ These trials excluded patients with severe left ventricular dysfunction (patients with an ejection fraction of $<35 \%$ ). A meta-analysis of the trials showed that $7.2 \%$ of the patients who underwent randomization had an ejection fraction of $40 \%$ or less, and only $4.0 \%$ had primary symptoms of heart failure rather than angina. ${ }^{9}$ Furthermore, these trials predate the major developments in medical therapy and cardiac surgery that have led to the current guidelines. ${ }^{10-13}$ More recently, observational analyses supporting a benefit of $\mathrm{CABG}^{14}$ and the proliferation of contemporary evidence-based medical and device-associated therapies have led to substantial clinical uncertainty regarding the incremental benefits of CABG relative to its risks in patients with ischemic cardiomyopathy. ${ }^{15,16}$

We designed the Surgical Treatment for Ischemic Heart Failure (STICH) trial to evaluate the role of cardiac surgery in the treatment of patients with coronary artery disease and left ventricular systolic dysfunction. A major hypothesis of the trial was that CABG plus intensive medical therapy based on current guidelines, as compared with medical therapy alone, would reduce mortality.

METHODS

\section{STUDY DESIGN}

The design of the STICH trial has been described previously. ${ }^{17,18}$ We conducted a multicenter, nonblinded, randomized study at 127 clinical sites in 26 countries. The trial protocol (available with the full text of this article at NEJM.org) was designed by several of the authors and was approved by the principal investigator and the eth- ics committee at each participating center. The trial was sponsored by the National Heart, Lung, and Blood Institute (NHLBI). Additional support was provided by Abbott Laboratories, which had no role in the conduct or reporting of the trial. An executive committee met weekly and monitored the daily conduct of the trial. An independent data and safety monitoring committee was appointed by the NHLBI. The Duke Clinical Research Institute coordinated all aspects of global trial operations, site management and monitoring, data collection, statistical analyses, and reporting. The authors reviewed the data, participated in the analyses and wrote the manuscript, and assume responsibility for the completeness and accuracy of the data and the analyses and for the fidelity of the study to the trial protocol.

\section{STUDY PATIENTS}

Eligible patients had coronary artery disease that was amenable to $\mathrm{CABG}$ and an ejection fraction of $35 \%$ or less, as determined at each enrolling site. Details of the enrollment criteria are provided in Table 1 in the Supplementary Appendix, available at NEJM.org. All patients provided written informed consent, as approved by the local institutional review board.

After initial determination of overall eligibility for the trial, patients were assessed to determine whether they were potential candidates for any of three possible therapeutic options: medical therapy alone, medical therapy plus CABG, or medical therapy plus CABG and surgical ventricular reconstruction. Patients were eligible for medical therapy alone if they did not have a stenotic lesion leading to loss of $50 \%$ or more of the diameter of the left main coronary artery and if they did not have Canadian Cardiovascular Society class III or IV angina while receiving medical therapy. (The Canadian Cardiovascular Society angina classification ranges from class 0 , which indicates no symptoms, to class IV, which indicates angina at any level of physical exertion.) Patients were eligible for surgical ventricular reconstruction if they had dominant anterior left ventricular akinesia or dyskinesia. As noted above, all patients were eligible for CABG.

On the basis of these eligibility criteria, patients were assigned by the enrolling physician to one of three trial strata. Stratum A included patients who were eligible for either medical therapy alone or medical therapy plus CABG, stratum B included patients who were eligible for any of the 
three treatment options, and stratum $\mathrm{C}$ included patients who were eligible for either medical therapy plus CABG or medical therapy plus CABG and surgical ventricular reconstruction (Fig. 1 in the Supplementary Appendix). Patients were then randomly assigned to one of the treatment options for which they were eligible. As a result, all the patients in stratum A and some of the patients in stratum $B$ were randomly assigned to either medical therapy alone or medical therapy plus CABG (the hypothesis 1 component of the STICH trial). All the patients in stratum $\mathrm{C}$ and some of the patients in stratum $B$ were randomly assigned to either medical therapy plus CABG or medical therapy plus $C A B G$ and surgical ventricular reconstruction (the hypothesis 2 component of the STICH trial). The results of the hypothesis 2 comparison have been reported previously. ${ }^{19}$ The results of the hypothesis 1 comparison are reported here.

\section{STUDY PROCEDURES}

During the initial evaluation, information was obtained on demographic factors and on clinical characteristics, including current medications and prior diagnostic and other cardiovascular procedures, and a physical examination was performed. Patients were randomly assigned to medical therapy alone or to medical therapy plus CABG by means of an investigator-initiated telephone call to an interactive voice-response system.

A lead cardiologist at each center was responsible for recommending the most appropriate medications and devices for the treatment of heart failure and coronary artery disease on the basis of current guidelines. Adherence to treatment guidelines was emphasized in the care of all patients and was monitored by a medical therapy committee. Cardiac surgery was performed by surgeons who had provided data on at least 25 patients with an ejection fraction of $40 \%$ or less in whom they had performed CABG and among whom the operative death rate was $5 \%$ or less. CABG was to be performed within 14 days after randomization. A surgical therapy committee monitored the conduct of surgery during the enrollment period. All patients underwent follow-up evaluations at the time of discharge or at 30 days, every 4 months for the first year, and every 6 months thereafter.

\section{STUDY OUTCOMES}

The primary outcome was the rate of death from any cause. Prespecified secondary outcomes in- cluded the rate of death from cardiovascular causes and the rate of death from any cause or hospitalization for cardiovascular causes. The causes of death and of selected secondary outcomes were adjudicated according to prespecified criteria by an independent clinical events committee whose members were unaware of the treatment assignments (see the definition of events in the Supplementary Appendix).

\section{STATISTICAL ANALYSIS}

We originally estimated that with a sample of 2000 patients who would be followed for an average of approximately 3 years, the study would have $90 \%$ power to detect a $25 \%$ reduction in mortality with CABG as compared with medical therapy alone, assuming a 3-year mortality of $25 \%$ in the medical-therapy group. Because enrollment was slower than expected, we modified the design so that the sample size was reduced and the duration of the follow-up period was increased correspondingly. The final study design specified that a sufficient number of patients had to be enrolled and the follow-up period had to be long enough that 400 deaths would occur; given this requirement, we estimated that we would need to enroll approximately 1200 patients, with an average follow-up period of 5 years. These numbers allowed for as much as a $20 \%$ treatment crossover from medical therapy to CABG without reducing the study's power to an unacceptable level. Randomization was performed with the use of permuted blocks and was stratified according to clinical site and stratum (A or B, as described above).

All major comparisons between the treatment groups were performed according to the intention-to-treat principle. Two-sided significance testing was used for all statistical tests. Cumulative event rates were calculated according to the Kaplan-Meier method, ${ }^{20}$ with all event or censoring times measured from the time of randomization. The significance of differences in mortality between the treatment groups was assessed with the use of the log-rank test, with adjustment for randomization stratum. Relative risks were expressed as hazard ratios with associated confidence intervals and were derived from the Cox proportional-hazards model. ${ }^{21,22}$ To characterize the time-dependent nature of the relative risks of the groups according to the treatment to which they had been randomly assigned, hazard ratios (and confidence intervals) were examined within time intervals of clinical importance: random- 


\begin{tabular}{|c|c|c|}
\hline Variable & $\begin{array}{l}\text { Medical Therapy } \\
(\mathrm{N}=602)\end{array}$ & $\begin{array}{c}\text { CABG } \\
(N=610)\end{array}$ \\
\hline \multicolumn{3}{|l|}{ Age $-y r$} \\
\hline Median & 59 & 60 \\
\hline Interquartile range & $53-67$ & $54-68$ \\
\hline Female sex - no. (\%) & $75(12)$ & $73(12)$ \\
\hline \multicolumn{3}{|l|}{ Race or ethnic group — no. $(\%) \dagger$} \\
\hline White & $402(67)$ & $389(64)$ \\
\hline Hispanic, Latino, or nonwhite & $200(33)$ & $221(36)$ \\
\hline \multicolumn{3}{|l|}{ Body-mass indextr } \\
\hline Median & 27 & 27 \\
\hline Interquartile range & $24-30$ & $24-30$ \\
\hline \multicolumn{3}{|l|}{ Medical history — no. (\%) } \\
\hline Previous myocardial infarction & $472(78)$ & $462(76)$ \\
\hline Hyperlipidemia & $370(61)$ & $360(59)$ \\
\hline Hypertension & $370(61)$ & $358(59)$ \\
\hline Diabetes & $238(40)$ & $240(39)$ \\
\hline Previous percutaneous coronary intervention & $74(12)$ & $82(13)$ \\
\hline Chronic renal insufficiency & $45(7)$ & $49(8)$ \\
\hline Previous stroke & $41(7)$ & $51(8)$ \\
\hline Previous CABG & $14(2)$ & $22(4)$ \\
\hline Current smoker & $122(20)$ & $130(21)$ \\
\hline \multicolumn{3}{|l|}{ Current CCS angina class $\rrbracket$} \\
\hline 0 & $225(37)$ & $217(36)$ \\
\hline I & $91(15)$ & $96(16)$ \\
\hline II & $260(43)$ & $265(43)$ \\
\hline III & $23(4)$ & $25(4)$ \\
\hline IV & $3(<1)$ & $7(1)$ \\
\hline \multicolumn{3}{|l|}{ Current NYHA class } \\
\hline 1 & $74(12)$ & $65(11)$ \\
\hline II & $307(51)$ & $319(52)$ \\
\hline III & $205(34)$ & $207(34)$ \\
\hline IV & $16(3)$ & $19(3)$ \\
\hline \multicolumn{3}{|l|}{ Systolic blood pressure - mm Hg } \\
\hline Median & 120 & 120 \\
\hline Interquartile range & $110-130$ & $110-130$ \\
\hline \multicolumn{3}{|l|}{ Pulse - beats/min } \\
\hline Median & 72 & 74 \\
\hline Interquartile range & $65-80$ & $66-82$ \\
\hline \multicolumn{3}{|l|}{ 6-Minute walk distance - $\mathrm{ft}$ q } \\
\hline Median & 1115 & 1145 \\
\hline Interquartile range & $840-1345$ & $863-1320$ \\
\hline
\end{tabular}

* CABG denotes coronary-artery bypass grafting, and NYHA New York Heart Association.

$\uparrow$ Race or ethnic group was self-reported.

7 The body-mass index is the weight in kilograms divided by the square of the height in meters.

$\int$ The Canadian Cardiovascular Society (CCS) angina classification ranges from class 0 , which indicates no symptoms, to class IV, which indicates angina at any level of physical exertion.

9 To convert the values for the 6-minute walk distance to meters, multiply by 0.305 . 


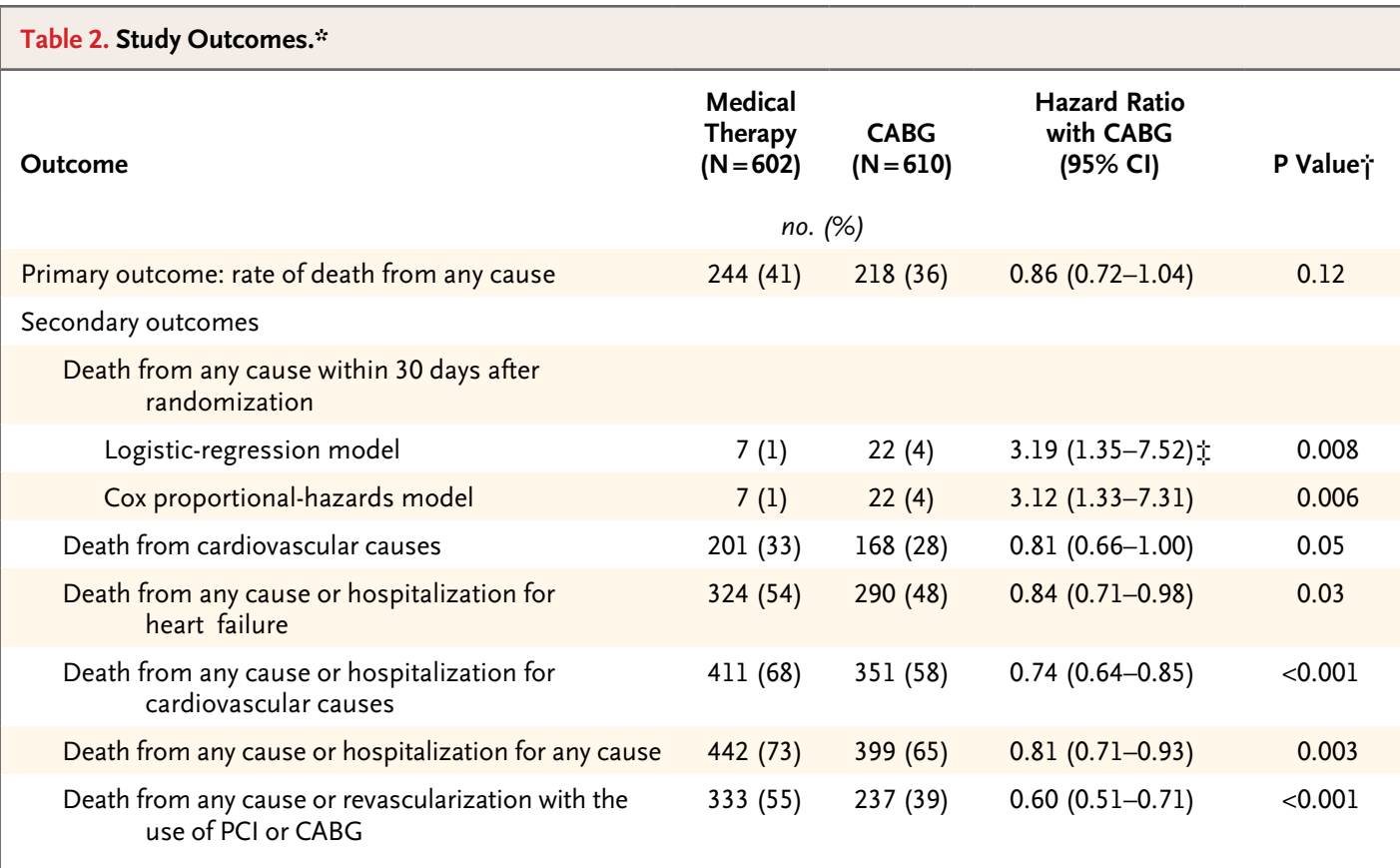

* All analyses were adjusted for patient stratum (stratum A, which included patients eligible for either medical therapy alone or medical therapy plus CABG vs. stratum B, which included patients eligible for medical therapy alone, medical therapy plus $C A B G$, or medical therapy plus $C A B G$ and surgical ventricular reconstruction). CABG denotes coronaryartery bypass grafting, and $\mathrm{PCI}$ percutaneous coronary intervention.

$\dagger$ All P values were calculated with the use of the log-rank test, except for one of the analyses of death from any cause within 30 days after randomization, for which, as noted, the $P$ value was calculated with the use of the logistic-regression model.

$\downarrow$ This value is an odds ratio rather than a hazard ratio.

ization through 30 days, 31 through 365 days, 366 days through 2 years, and any time after 2 years. As prespecified in the study protocol, comparisons of the treatment groups were also performed with adjustment for key baseline factors (in addition to randomization stratum). The Cox model was also used to assess the consistency of treatment effects by testing for interactions between treatment and prespecified baseline characteristics.

To aid in an assessment of the effect of treatment crossovers, secondary as-treated and perprotocol analyses were also performed. The astreated comparison was analyzed with the Cox model in which CABG was treated as a timedependent covariate.

Ten interim analyses of the data were performed and were reviewed by the independent data and safety monitoring committee. Interim treatment comparisons for the primary outcome were monitored with the use of two-sided symmetric O'Brien-Fleming boundaries generated with the Lan-DeMets alpha-spending-function approach to group-sequential testing. ${ }^{23,24} \mathrm{~A}$ significance level of 0.04 was required for the primary outcome at the final analysis to adjust for the interim analyses. The final clinical assessment for each patient was performed in the 4-month period leading up to November 30, 2010, which was the cutoff date for analyses of all reported outcomes.

\section{RESULTS}

\section{STUDY POPULATION}

Between July 24, 2002, and May 5, 2007, a total of 2136 patients were enrolled in the overall STICH program; 1212 of these patients at 99 centers in 22 countries were enrolled in the hypothesis 1 component of the trial and were randomly assigned to receive medical therapy alone (602 patients) or medical therapy plus CABG (610 patients). Baseline demographic characteristics, clinical characteristics including details of ventricular function and coronary anatomy, and medication use were well balanced between the two groups (Table 1, and Tables 2 and 3 in the Supplementary Appendix). 


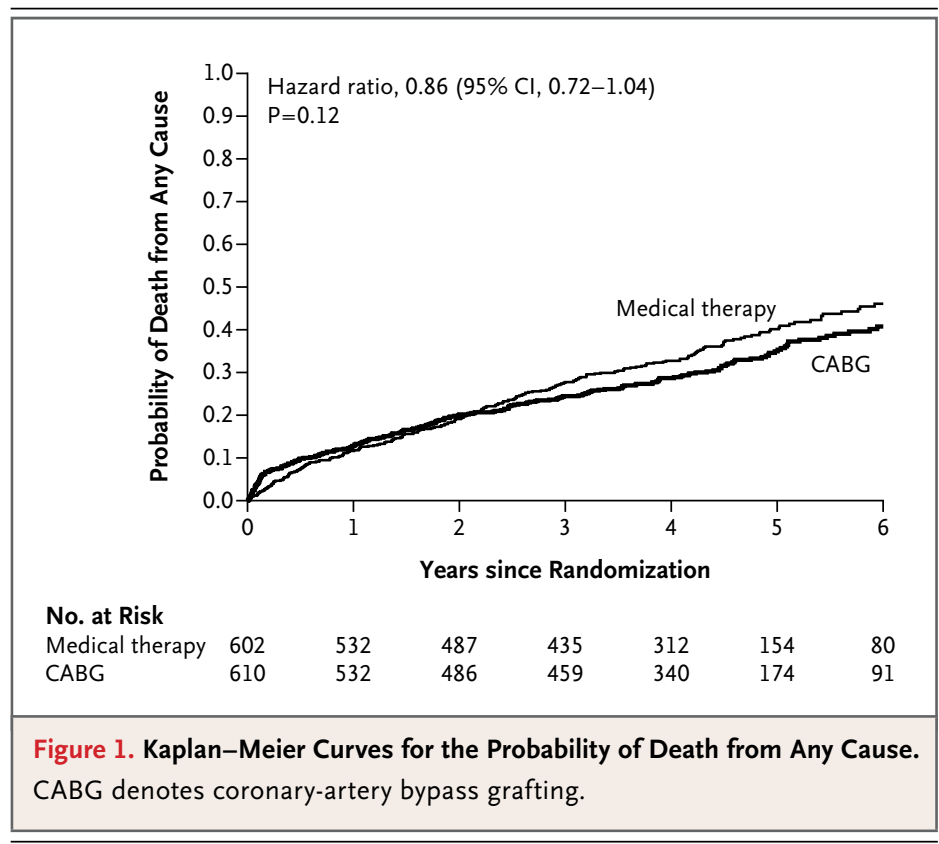

STUDY TREATMENTS

Of the 610 patients randomly assigned to CABG, 555 (91\%) underwent CABG before the end of the study; the median time to the CABG procedure was 10 days (interquartile range, 5 to 16), with a maximum of 177 days. The surgery was performed electively in 529 of these patients (95\%) and with urgency in 26 patients $(5 \%)$. The median time in the intensive care unit was 52.4 hours (interquartile range, 40.8 to 94.6 ), the median duration of intubation was 16.5 hours (interquartile range, 11.1 to 22.4), and the median length of stay in the hospital was 9.0 days (interquartile range, 7.0 to 13.0). A concurrent mitral-valve operation was performed in 63 patients (11\%). Of the 553 patients in the CABG group who underwent CABG and for whom data on arterial and venous conduits were available, $505(91 \%)$ received at least one arterial conduit and $473(86 \%)$ received one or more venous conduits. Overall, 484 patients $(87 \%)$ in the CABG group who underwent CABG had two or more distal anastomoses placed during surgery.

Of the 602 patients randomly assigned to medical therapy alone, $100(17 \%)$ underwent CABG before the end of the follow-up period. The median time to the CABG procedure was 142 days (interquartile range, 19 to 576), with a maximum of 2402 days. The most common indication for crossover to CABG was progressive symptoms $(40 \%)$, followed by acute decompensation ( $27 \%)$, patient's or family's decision (28\%), and physician's decision $(5 \%)$.
Adherence to guideline-based use of medications was high throughout the study period, without significant differences between the treatment groups (Table 3 in the Supplementary Appendix). Postrandomization procedures are summarized in Table 4 in the Supplementary Appendix.

\section{FOLLOW-UP}

Final follow-up status was ascertained between August 1, 2010, and November 30, 2010, for 1207 of the 1212 patients (99.6\%) who underwent randomization. During this last follow-up period, 5 patients could not be evaluated; the median time from randomization to the date of the last contact for these 5 patients, which was the date on which follow-up data were censored, was 40 months. The median length of follow-up was 56 months (interquartile range, 48 to 68), with a minimum of 12 months and a maximum of 100 months.

\section{OUTCOMES}

The primary outcome occurred in 244 of the 602 patients (41\%) assigned to medical therapy alone and in 218 of the 610 patients (36\%) assigned to CABG (hazard ratio with CABG, 0.86 ; $95 \%$ confidence interval [CI], 0.72 to $1.04 ; \mathrm{P}=0.12$ ) (Table 2 and Fig. 1). A total of 201 patients (33\%) assigned to medical therapy and 168 (28\%) assigned to CABG died from a cardiovascular cause (hazard ratio with CABG, $0.81 ; 95 \% \mathrm{CI}, 0.66$ to 1.00 ; $\mathrm{P}=0.05$ ) (Table 2 and Fig. 2A). Death from any cause or hospitalization for cardiovascular causes occurred in 411 patients $(68 \%)$ in the medicaltherapy group and 351 (58\%) in the CABG group (hazard ratio with CABG, 0.74; 95\% CI, 0.64 to 0.85; $\mathrm{P}<0.001$ ) (Table 2 and Fig. 2B). The results with respect to all other secondary clinical outcomes also favored CABG, except for 30-day mortality (Table 2). The results of models adjusted for covariates, including those with CABG as a timedependent covariate, are shown in Table 5 in the Supplementary Appendix.

We performed an as-treated analysis comparing the 592 patients who were treated with medical therapy throughout the first year after randomization and the 620 patients who underwent CABG either because they were randomly assigned to $\mathrm{CABG}$ or because they crossed over to CABG during year 1 of the follow-up period; the hazard ratio for the primary outcome with CABG was 0.70 (95\% CI, 0.58 to $0.84 ; \mathrm{P}<0.001$ ) (Table 5 and Fig. 2 in the Supplementary Appen- 
dix). We also performed a per-protocol comparison of the 537 patients randomly assigned to medical therapy who did not cross over to CABG during the first year of follow-up and the 555 patients assigned to CABG who actually underwent CABG; the hazard ratio for the primary outcome with CABG was 0.76 (95\% CI, 0.62 to 0.92; $\mathrm{P}=0.005$ ) (Table 5 and Fig. 3 in the Supplementary Appendix).

\section{SUBGROUP ANALYSES}

There were no significant interactions between treatment assignment and baseline characteristics of interest with respect to the primary outcome, as shown in the hazard-ratio plots in Figure 3. A nominally significant interaction with trial stratum was noted, but it is likely that this represents a chance finding.

\section{DISCUSSION}

We compared the strategy of medical therapy alone with that of medical therapy plus CABG in patients with coronary artery disease and left ventricular dysfunction. The intention-to-treat analysis showed that there was no significant difference between the two trial groups with respect to the primary outcome, the rate of death from any cause. Betweengroup differences in favor of CABG were seen with respect to the rate of death from cardiovascular causes and the rate of death from any cause or hospitalization for cardiovascular causes in the intention-to-treat analysis. The results of analyses performed according to whether patients actually underwent surgery, with and without adjustment for baseline variables, also favored CABG.

Although the randomization strategy specified in our statistical plan was adhered to, clinical circumstances led to crossover in the case of $17 \%$ of patients who had been randomly assigned to medical therapy and 9\% who had been randomly assigned to CABG. Per-treatment analyses suggest that this imbalance in crossover rates between groups modified the results of the primary intention-to-treat analysis by diminishing the treatment effect of CABG relative to medical therapy. Although we believe that these results are informative, since they mirror real-world clinical decision making, in which the choice of CABG is not influenced by randomization, these per-treatment analyses must be interpreted conservatively.

As anticipated, CABG was associated with an early risk of death from any cause among all pa-

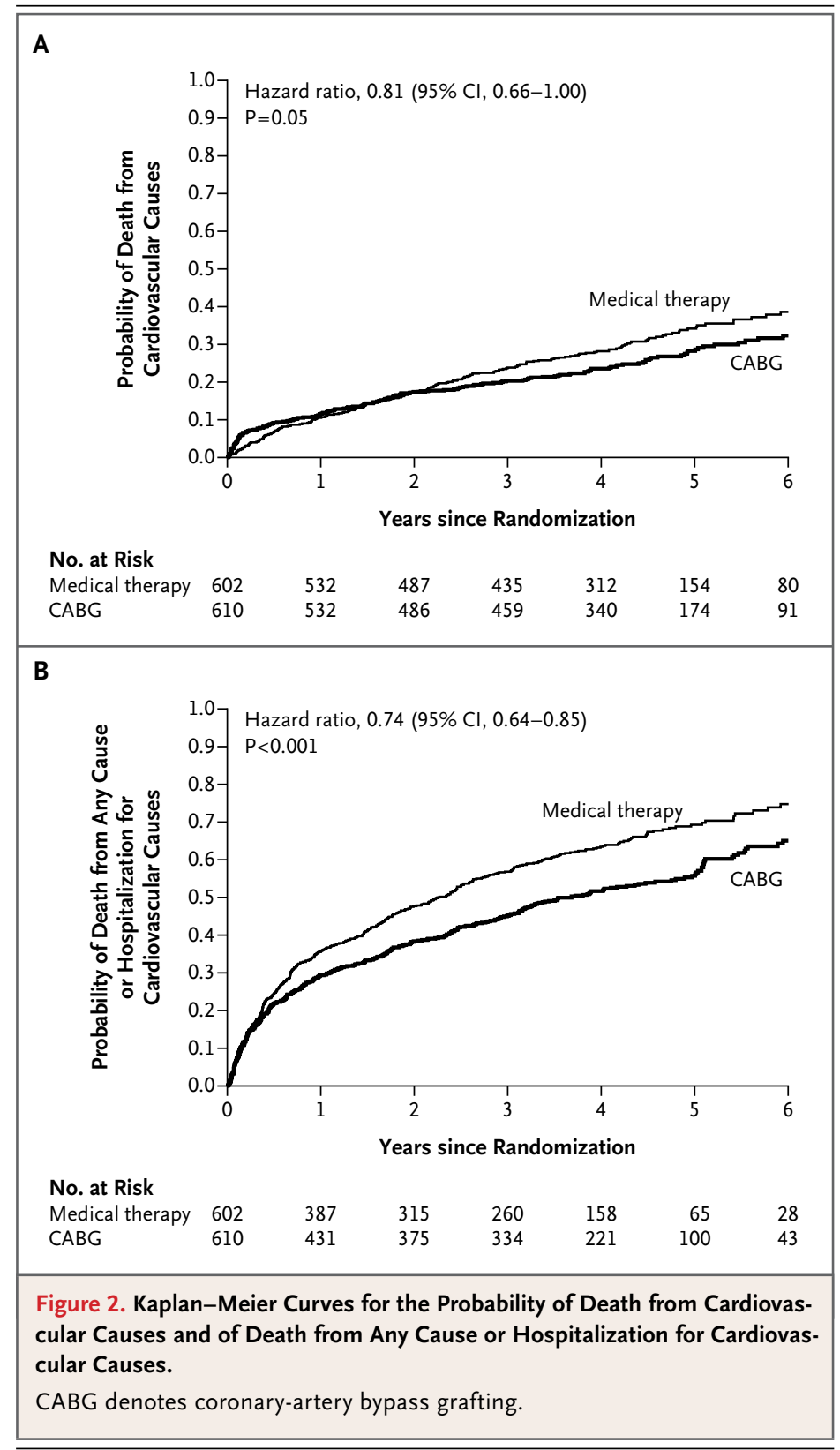

tients who underwent the procedure. When patients are treated with CABG and intensive medical therapy for coronary artery disease and left ventricular dysfunction, they are exposed to an early risk as a result of the surgical intervention. The total number of deaths was higher in the surgical group than in the medical-therapy group for more than 2 years after randomization.

Our study had several limitations. First, when the analysis in any trial fails to detect a significant difference between treatment groups with respect to the primary outcome, analyses of sec- 


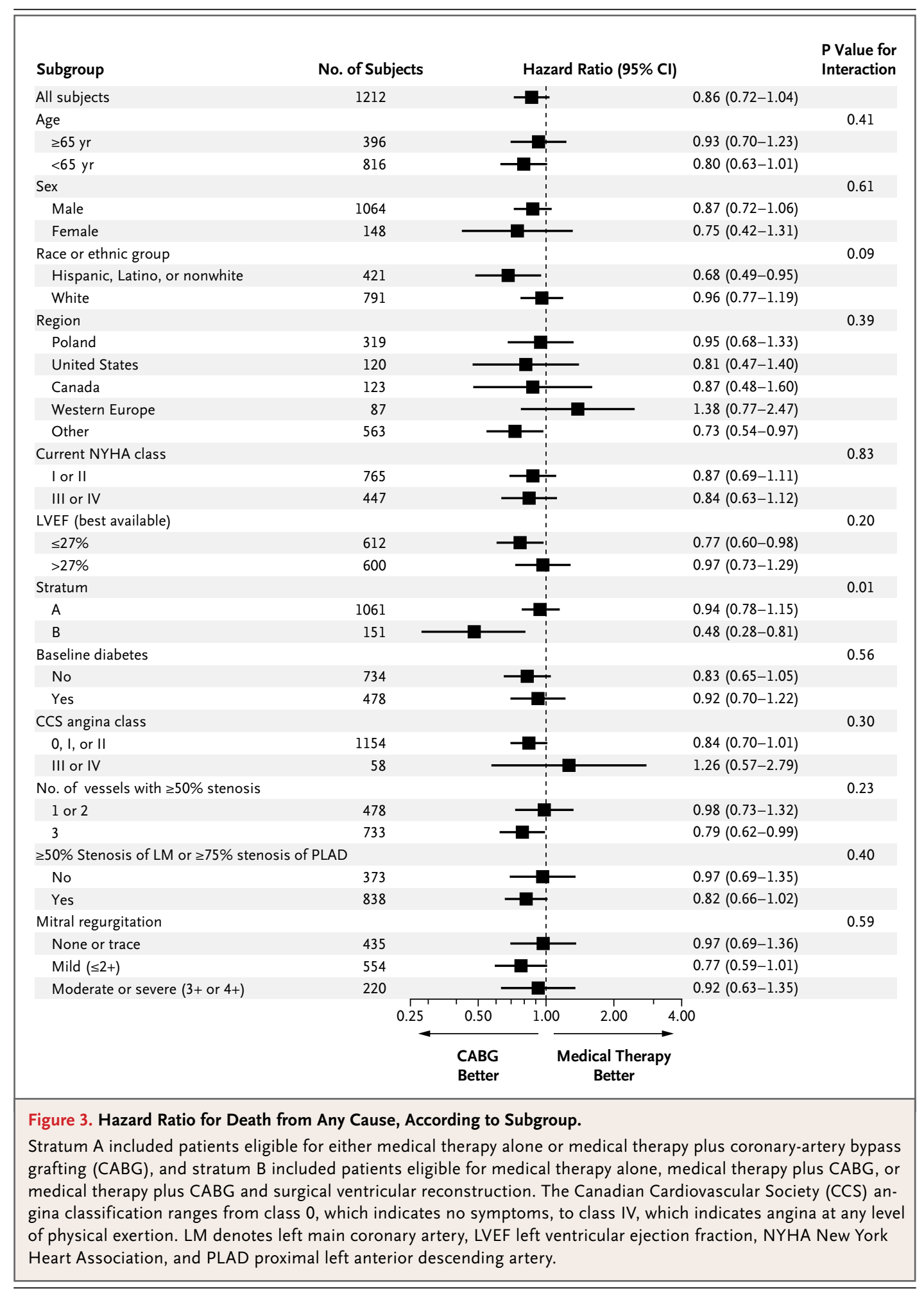

ondary outcomes showing a benefit must inevi- is presumably the mortality outcome most likely tably be considered to be somewhat provisional. to be influenced by CABG, had a P value of 0.05 , Second, the between-group difference with re- which would not have been significant if it had spect to death from cardiovascular causes, which been adjusted for multiple testing. Given the re- 
sults of other secondary analyses, we believe that the lack of an unequivocally significant betweengroup difference in the outcome of death from any given cause was more likely to have been due to limited power and limited duration of follow-up than to a true lack of benefit of CABG. Finally, since the trial was not blinded (and could not realistically have been blinded), the nonfatal outcomes (e.g., hospitalization due to various causes) could have been influenced by the clinician's knowledge of the treatment the patient received.

Clinical decision making for patients with coronary artery disease associated with left ventricular dysfunction has been constrained by the absence of randomized, comparative data on revascularization. In fact, small subgroup analyses of the previous randomized trials of CABG involving patients with chronic stable angina dissuaded many clinicians from pursuing diagnostic assessments for coronary artery disease or revascularization in patients presenting with heart failure. ${ }^{8,9}$ This approach continues to be supported by guidelines. ${ }^{10-13}$ In the absence of direct evidence, attention has shifted toward the detection of acutely and chronically underperfused but viable myocardium (i.e., myocardium that exhibits stunning or hibernation), a condition for which functional recovery may be achieved with reperfusion. ${ }^{25}$ Imaging strategies ${ }^{15,26}$ and clinical factors are routinely used to select patients who have the highest likelihood of functional recovery, as well as those in whom CABG should be avoided. In another report in this issue of the Journal, STICH investigators provide additional information on the interaction between viable myocardium and treatment effect. ${ }^{27}$

In summary, the STICH trial compared medical therapy alone with medical therapy plus CABG in patients with coronary artery disease and left ventricular dysfunction. There was no significant difference between the two study groups with respect to the primary end point of the rate of death from any cause. The rates of death from cardiovascular causes and of death from any cause or hospitalization for cardiac causes were lower among patients assigned to CABG than among those assigned to medical therapy.

\footnotetext{
Supported by grants (U01HL69015 and U01HL69013) from the National Heart, Lung, and Blood Institute and by Abbott Laboratories.

Dr. Velazquez reports receiving consulting fees from Novartis, Gilead, and Boehringer Ingelheim Pharmaceuticals; Dr. Abraham, consulting fees from Medtronic, St. Jude Medical, Biotronik, and CardioMEMS; and Dr. Rouleau, consulting fees and grant support from Novartis. No other potential conflict of interest relevant to this article was reported.

Disclosure forms provided by the authors are available with the full text of this article at NEJM.org.

We thank Vanessa Moore, Doreen Bain, Anthony Doll, and Elizabeth Cook of the Duke Clinical Research Institute for their assistance with the preparation of the manuscript, and all the patients in the STICH trial for their participation in the study.
}

\section{APPENDIX}

The authors' affiliations are as follows: Division of Cardiovascular Medicine (E.J.V., C.M.O.), Departments of Biostatistics and Bioinformatics (K.L.L.) and Surgery (R.H.J.), and Duke Clinical Research Institute (L.S., G.R.R.), Duke University Medical Center, Durham, NC; Second Department of Cardiac Surgery, Medical University of Silesia, Katowice (M.A.D.), and National Institute of Cardiology, Warsaw (H.S.) - both in Poland; SAL Hospital and Medical Institute, Ahmedabad (A.J.), and Center for Chronic Disease Control, New Delhi (D.P.) - both in India; Division of Cardiovascular Sciences, National Heart, Lung, and Blood Institute, Bethesda, MD (G.S.); Research Institute of Circulation Pathology, Novosibirsk, Russia (A.M.); Division of Cardiac Surgery, Dalhousie University and the Queen Elizabeth II Health Sciences Centre, Halifax, NS, Canada (I.S.A.); Keck School of Medicine, Viterbi School of Engineering, University of Southern California, Los Angeles (G.P.); Dedinje Cardiovascular Institute, University of Belgrade School of Medicine, Belgrade, Serbia (S.G.); Division of Cardiovascular Medicine, Ohio State University, Columbus (W.T.A.); Departments of Cardiothoracic Surgery and Surgery, University of Melbourne, St. Vincent's Hospital, Melbourne, VIC, Australia (M.Y.); Dipartimento Cardiovascolare Clinico e di Ricerca, Ospedali Riuniti Bergamo, Bergamo, Italy (P.F.); Scottish National Advanced Heart Failure Service, Golden Jubilee National Hospital, Glasgow, United Kingdom (M.C.P.); Siriraj Hospital, Mahidol University, Bangkok, Thailand (P.P.); Division of Cardiology, Northwestern University, Chicago (R.O.B.); and Montreal Heart Institute, Montreal (J.-L.R.).

REFERENCES

1. Lloyd-Jones D, Adams RJ, Brown TM, et al. Heart disease and stroke statistics - 2010 update: a report from the American Heart Association. Circulation 2010; 121(7):e46-e215.

2. Dickstein K, Cohen-Solal A, Filippatos $\mathrm{G}$, et al. ESC guidelines for the diagnosis and treatment of acute and chronic heart failure 2008: the Task Force for the Diagnosis and Treatment of Acute and Chronic Heart Failure 2008 of the European Society of Cardiology: developed in collabora- tion with the Heart Failure Association of the ESC (HFA) and endorsed by the European Society of Intensive Care Medicine (ESICM). Eur Heart J 2008;29:2388-442.

3. Gheorghiade M, Sopko G, De Luca L, et al. Navigating the crossroads of coronary artery disease and heart failure. Circulation 2006;114:1202-13.

4. Coronary Artery Surgery Study (CASS): a randomized trial of coronary artery bypass surgery: survival data. Circulation 1983;68:939-50.
5. The Veterans Administration Coronary Artery Bypass Surgery Cooperative Study Group. Eleven-year survival in the Veterans Administration randomized trial of coronary bypass surgery for stable angina. N Engl J Med 1984;311:1333-9. 6. Varnauskas E. Twelve-year follow-up of survival in the randomized European Coronary Surgery Study. N Engl J Med 1988;319:332-7.

7. Passamani E, Davis KB, Gillespie MJ, Killip T. A randomized trial of coronary 
artery bypass surgery: survival of patients with a low ejection fraction. $N$ Engl J Med 1985;312:1665-71.

8. Alderman EL, Fisher LD, Litwin P, et al. Results of coronary artery surgery in patients with poor left ventricular function (CASS). Circulation 1983;68:785-95.

9. Yusuf S, Zucker D, Peduzzi P, et al. Effect of coronary artery bypass graft surgery on survival: overview of 10 -year results from randomised trials by the Coronary Artery Bypass Graft Surgery Trialists Collaboration. Lancet 1994;344:563-70. [Erratum, Lancet 1994;344:1446.]

10. Hunt SA, Abraham WT, Chin MH, et al. 2009 Focused update incorporated into the ACC/AHA 2005 Guidelines for the Diagnosis and Management of Heart Failure in Adults: a report of the American College of Cardiology Foundation/ American Heart Association Task Force on Practice Guidelines developed in collaboration with the International Society for Heart and Lung Transplantation. J Am Coll Cardiol 2009;53(15):e1-e90. [Erratum, J Am Coll Cardiol 2009;54:2464.]

11. Eagle KA, Guyton RA, Davidoff R, et al. ACC/AHA 2004 guideline update for coronary artery bypass graft surgery: a report of the American College of Cardiology/American Heart Association Task Force on Practice Guidelines (Committee to Update the 1999 Guidelines for Coronary Artery Bypass Graft Surgery). Circulation 2004;110(14):e340-437. [Erratum, Circulation 2005;111:2014.]

12. Fraker TD Jr, Fihn SD, Gibbons RJ, et al. 2007 Chronic angina focused update of the ACC/AHA 2002 guidelines for the management of patients with chronic stable angina: a report of the American College of Cardiology/American Heart Association Task Force on Practice Guidelines Writing Group to develop the focused update of the 2002 guidelines for the management of patients with chronic stable angina. J Am Coll Cardiol 2007; 50:2264-74.

13. Wijns W, Kolh P, Danchin N, et al. Guidelines on myocardial revascularization: the Task Force on Myocardial Revascularization of the European Society of Cardiology (ESC) and the European Association for Cardio-Thoracic Surgery (EACTS). Eur Heart J 2010;31:2501-55.

14. O'Connor CM, Velazquez EJ, Gardner LH, et al. Comparison of coronary artery bypass grafting versus medical therapy on long-term outcome in patients with ischemic cardiomyopathy (a 25-year experience from the Duke Cardiovascular Disease Databank). Am J Cardiol 2002;90:101-7.

15. Cleland JG, Calvert M, Freemantle N, et al. The Heart Failure Revascularisation Trial (HEART). Eur J Heart Fail 2011; 13:227-33.

16. Chareonthaitawee P, Gersh BJ, Araoz PA, Gibbons RJ. Revascularization in severe left ventricular dysfunction: the role of viability testing. J Am Coll Cardio 2005;46:567-74

17. Velazquez EJ, Lee KL, O'Connor CM, et al. The rationale and design of the Surgical Treatment for Ischemic Heart Failure (STICH) trial. J Thorac Cardiovasc Surg 2007;134:1540-7.
18. Jones RH, White H, Velazquez EJ, et al. STICH (Surgical Treatment for Ischemic Heart Failure) trial enrollment. J Am Coll Cardiol 2010;56:490-8.

19. Jones RH, Velazquez EJ, Michler RE, et al. Coronary bypass surgery with or without surgical ventricular reconstruction. N Engl J Med 2009;360:1705-17.

20. Kaplan EL, Meier P. Nonparametric estimation from incomplete observations. J Am Stat Assoc 1958;53:457-81.

21. Cox DR. Regression models and lifetables. J R Stat Soc [B] 1972;34:187-220.

22. Breslow N. Covariance analysis of censored survival data. Biometrics 1974; 30:89-99.

23. Lan KKG, DeMets DL. Discrete sequential boundaries for clinical trials. Biometrika 1983;70:659-63.

24. O'Brien PC, Fleming TR. A multiple testing procedure for clinical trials. Biometrics 1979;35:549-56.

25. Braunwald E, Rutherford JD. Reversible ischemic left ventricular dysfunction: evidence for the "hibernating myocardium." J Am Coll Cardiol 1986;8:1467-70.

26. Beanlands RS, Nichol G, Huszti E, et al. F-18-fluorodeoxyglucose positron emission tomography imaging-assisted management of patients with severe left ventricular dysfunction and suspected coronary disease: a randomized, controlled trial (PARR-2). J Am Coll Cardiol 2007;50:2002-12.

27. Bonow RO, Maurer G, Lee KL, et al. Myocardial viability and survival in ischemic left ventricular dysfunction. $\mathrm{N}$ Engl J Med 2011;364:1617-25.

Copyright (๑) 2011 Massachusetts Medical Society.

AN NEJM APP FOR IPHONE

The NEJM Image Challenge app brings a popular online feature to the smartphone. Optimized for viewing on the iPhone and iPod Touch, the Image Challenge app lets you test your diagnostic skills anytime, anywhere. The Image Challenge app randomly selects from 300 challenging clinical photos published in NEJM, with a new image added each week. View an image, choose your answer, get immediate feedback, and see how others answered.

The Image Challenge app is available at the iTunes App Store. 